$\mathrm{DE}$

M E D I C I N A

T R O P I C A L

$\mathrm{DE}$

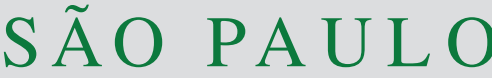

JOURNAL OF THE SÃO PAULO INSTITUTE OF TROPICAL MEDICINE

'Universidade Federal do Ceará, Faculdade de Medicina, Departamento de Patologia e Medicina Legal, Fortaleza, Ceará, Brazil

2Instituto do Câncer do Ceará, Hospital Haroldo Juaçaba, Fortaleza, Ceará, Brazil

${ }^{3}$ Hospital Infantil Albert Sabin, Fortaleza, Ceará, Brazil

Correspondence to: Sami de Andrade Cordeiro Gadelha

Universidade Federal do Ceará, Faculdade de Medicina, Departamento de Patologia e Medicina Legal, Rua Monsenhor Furtado, Rodolfo Teófilo, CEP 60441-750, Fortaleza, CE, Brazil

Tel: +55 8533668301

Fax: +558533668300

E-mail: samigadelha@gmail.com

Received: 28 November 2018

Accepted: 2 April 2019

\section{Evaluation of the diagnostic potential of CD1a immunohistochemistry for visceral leishmaniasis}

Sami de Andrade Cordeiro Gadelha1, Maria do Perpétuo Socorro Saldanha da Cunha ${ }^{2,3}$, Gabriela Maia Coelho', Tamises Melo Siqueira Marinho', Carlos Gustavo Hirth ${ }^{1,2,3}$

\section{ABSTRACT}

Visceral Leishmaniasis is a public health problem caused by protozoans of the genus Leishmania. K39 serological test is commonly used in the initial investigation, with high specificity, but variable sensitivity. Amastigotes can be identified by optical microscopy, however, the differential diagnosis with cellular debris or other intracellular parasites is necessary. Recent studies have raised the possibility of using immunohistochemistry in the diagnosis of visceral leishmaniasis with labeling of amastigotes by the anti-CD1a antibody. This retrospective study was based on 38 samples from patients with visceral leishmaniasis whose diagnoses were confirmed by myelogram and/or k39 testing, aside from positive $(\mathrm{N}=13)$ and negative biopsies $(\mathrm{N}=25), 2$ samples from patients with false positive biopsies for visceral leishmaniasis and 8 samples from patients with histoplasmosis diagnosis. The histological slides were evaluated for the presence of amastigotes and their Modified Ridley Parasitic Index. The samples were submitted to immunohistochemical reactions using the anti-CD1a antibody with MTB1 and O10 clones. Immunohistochemical reactions with MTB1 and O10 clones had low sensitivity in this study. However, all bone marrow samples were previously decalcified with nitric acid which is probably a deleterious treatment for immunohistochemical reactions in this site. Excluding these samples, we obtained $58.33 \%$ sensitivity and $100 \%$ specificity with the MTB1 clone. Despite the intermediate sensitivity, the immunohistochemistry for the CD1a marker with clone MTB1 can be useful in the differential diagnosis of visceral leishmaniasis, helping to discriminate leishmania amastigotes from other pathogens with similar morphology and cellular debris in different samples, except in bone marrow biopsies previously decalcified with nitric acid.

KEYWORDS: Leishmaniasis. Leishmania. Biopsy. Immunohistochemistry.

\section{INTRODUCTION}

Visceral leishmaniasis is a growing public health problem in America, Asia, Europe and Africa. Its importance led the World Health Organization (WHO) to include it among the six priority diseases in the control program of this institution ${ }^{1}$. According to the Brazilian Ministry of Health, in 19 years of notification (19842002 ), visceral leishmaniasis totaled 48,455 cases and approximately $66 \%$ occurred in the Bahia, Ceara, Maranhao and Piaui States ${ }^{2}$. The etiological agents of visceral leishmaniasis are protozoans of the genus Leishmania, exclusively intracellular parasites of the mononuclear phagocytic system cells, with a flagellate or promastigote form found in the digestive tract of the insect vector and an aflagellate or amastigote form within vertebrate tissues ${ }^{3}$. Most infections are clinically 
diagnosed. Patients usually presents with fever, cutaneousmucosal pallor, hepatosplenomegaly andpancytopenia ${ }^{3}$.

Serological tests are the most commonly used complementary diagnostic methods ${ }^{3}$. The rapid rK39 antigen-based immunochromatographic test Kalazar Detect(r), or K39 test, is a qualitative test of serum antibodies against the recombinant antigen K39 of Leishmania chagasi species, has high specificity (100\%), however its sensitivity is variable in different countries ${ }^{4}$. In addition, one can identify parasites of the genus Leishmania by optical microscopy ${ }^{3}$. Peripheral blood smears are easily collected, however, there are few circulating parasite cells ${ }^{3}$. Myelogram, a bone marrow aspirate analysis, is a frequently used method ${ }^{3}$. Direct visualization of amastigotes by biopsies of bone marrow, liver, lymph node and other visceral organs is a valid method, especially in cases with high parasitic load ${ }^{3}$. In biopsies, the differential diagnosis between amastigotes and cell debris within macrophages is necessary as it is a common finding in hemophagocytic syndromes and in native neoplasms or metastases with bone marrow involvement ${ }^{5}$, clinical conditions potentially mimicking visceral leishmaniasis ${ }^{6}$. Another challenge for the morphological diagnosis of amastigotes by optical microscopy is to differentiate them from other intracellular parasites such as the Histoplasma capsulatum fungus which has similar morphology and involvement of lymph nodes, liver, spleen and bone marrow ${ }^{7}$. Recent studies have raised the possibility of using immunohistochemistry to aid in the parasitological diagnosis of leishmaniasis with the labeling of amastigotes by the anti-CD1a antibody, clone MTB1, but mainly in cutaneous leishmaniasis, with few studies exploring this possibility in the context of visceral leishmaniasis. A study has demonstrated this in all 19 cases of cutaneous leishmaniasis caused by Leishmania infantum, in addition to finding positivity in all four cases of visceral leishmaniasis ${ }^{8}$. However, the small number of visceral leishmaniasis samples tested in this study is noteworthy.

The CD1a antigen is a transmembrane glycoprotein present in the plasma membrane of dendritic cells and thymocytes, structurally related to molecules of the Major Histocompatibility Complex (MHC) $)^{9}$. Some hypotheses have been proposed to justify the labeling of amastigotes by an antibody against a dendritic cell surface antigen. Among them, the most accepted is that amastigotes acquire the CD1a surface antigen after phagocytosis by dendritic cells, exposing CD1a in its own surface and kinetoplast ${ }^{10}$. This hypothesis is reinforced by the finding that samples from culture media of leishmania promastigotes do not express CD1a, as opposed to cases of cutaneous leishmaniasis caused by Leishmania major amastigotes, which are positive for this marker ${ }^{11}$. Immunohistochemical markers developed for specific antigens of Leishmania sp are not commercially available, so that diagnostic centers have to develop their own markers, increasing costs due to standardization and all necessary procedures to minimize the risk of cross reactions with other protozoa belonging to the Trypanosomatidae family.

Considering all the above mentioned, the use of a commercially available immunohistochemical marker as the anti-CD1a, an antibody widely used for other purposes such as the diagnosis of histiocytosis, could represent an enormous gain for different medical centers, even those not focused on research or diagnosis of infectious diseases.

\section{MATERIAL AND METHODS}

The present study was retrospective and based on biopsy specimens collected between 2006 and 2018 from patients diagnosed with visceral leishmaniasis or histoplasmosis, considering results obtained in biopsies, and/or myelograms or the K39 test, attended at Hospital Infantil Albert Sabin, Fortaleza, Ceara State; at Hospital Haroldo Juaçaba/ Instituto do Cancer do Ceara, Fortaleza, Ceara State; and at Hospital Universitario Walter Cantidio, Universidade Federal do Ceara, Fortaleza, Ceara State. This research was approved by the Research Ethics Committee of Hospital Universitario Walter Cantidio (protocol $N^{\circ} 2.909 .806$ ). Medical notes of patients from the participating institutions with suspected but not conclusive diagnosis of visceral leishmaniasis or histoplasmosis were included in the study, provided that there was enough archived biopsy material, from bone marrow and other sites, suitable for immunohistochemical reactions. Patients without archived tissue or with insufficient tissue for analysis were excluded. We obtained a total of 48 cases.

Regarding the biopsy sites included in this study, a pathology laboratory receives various organs from patients under clinical investigation for a variety of conditions, and, sometimes, the diagnosis of visceral leishmaniasis was made by biopsies other than those from bone marrow.

Medical records from patients were reviewed to evaluate clinical and serological diagnosis, treatment and response to treatment. The histological slides were reviewed for the presence or absence of amastigotes, associated with the measurement of Modified Ridley Parasitic Index, based on the number of parasites permicroscopic field (40x magnification): score 6 , more than 100,000 parasites; score 5, between 10,000 and 100,000 parasites; score 4, between 1,000 and 10,000 parasites; score 3, between 100 and 1,000 parasites; score 2, between 10 and 100 parasites; score 1 , between 1 and 10 parasites; score 0, zero parasites $^{12}$. Slides of all samples were stained by PAS and 
Grocott's methenamine silver staining to exclude deep mycosis. After slide revision, special staining evaluation and revision of medical records, the patients were divided into four groups:

True positives - 13 cases with samples containing amastigotes in histopathology, negative results for mycosis according to PAS and Grocott's methenamine silver staining.

False positives - 2 cases that were suggestive of visceral leishmaniasis by biopsy, with visualization of cellular debris within macrophages, but with negative myelogram and $\mathrm{K} 39$ test results.

True negatives - 8 cases with samples containing amastigotelike structures with positive staining by PAS and/or Grocott's methenamine silver staining, characterizing Histoplasma capsulatum yeast cells.

False negatives - 25 cases with myelogram positive samples for amastigotes, but with negative biopsy samples, as well as patients with K39 positive results but negative by direct microscopy who responsed to therapy according to medical records.

Immunohistochemical reactions were performed using anti-CD1a, MTB1 (Cell Marque/Merk KGaA, Darmstadt, Germany) and O10 (DAKO/Agilent Technologies Inc., Santa Clara, CA, USA) antibody clones, after dewaxing and tissue treatment with the epitope recovery solutions PT Link ${ }^{\mathrm{TM}}$, following the manufacturer's recommendations ((DAKO/Agilent Technologies Inc., Santa Clara, CA, USA).
Samples were incubated with the Auto Stainer Link $48^{\mathrm{TM}}$ (DAKO/Agilent Technologies Inc., Santa Clara, CA, USA) and visualized using the EnVision Flex ${ }^{\mathrm{TM}}$ polymer (DAKO/Agilent Technologies Inc., Santa Clara, CA, USA). All reactions were performed with positive and negative controls. Positive controls were external samples containing dendritic cells from skin tissue and from a lymph node with Langerhans cell histiocytosis. Negative controls were internal, constituted by fragments of the samples known not to express CD1a. Sensitivity, specificity and accuracy of the CD1a marker were calculated for the diagnosis of visceral leishmaniasis.

\section{RESULTS}

In immunohistochemical positive reactions amastigotes were labeled in a pattern of peripheral membrane-like reinforcement, leaving the unstained nucleus in the center with a reinforcement in one pole, apparently because of the kinetoplast staining ${ }^{8}$ (Figure 1).

Regarding the anatomical site and the Modified Ridley Parasitic Index, the results of immunohistochemical reactions are summarized in Table 1. The CD1a immunohistochemical reactions had low sensitivity in this study, 23.68\% for MTB1 clone and $2.63 \%$ for $\mathrm{O} 10$ clone. However, negative results were obtained in almost all bone marrow samples for both MTB1 (26 of 28) and O10 (27 of 28). When considering biopsy specimens from other sites, excluding bone marrow

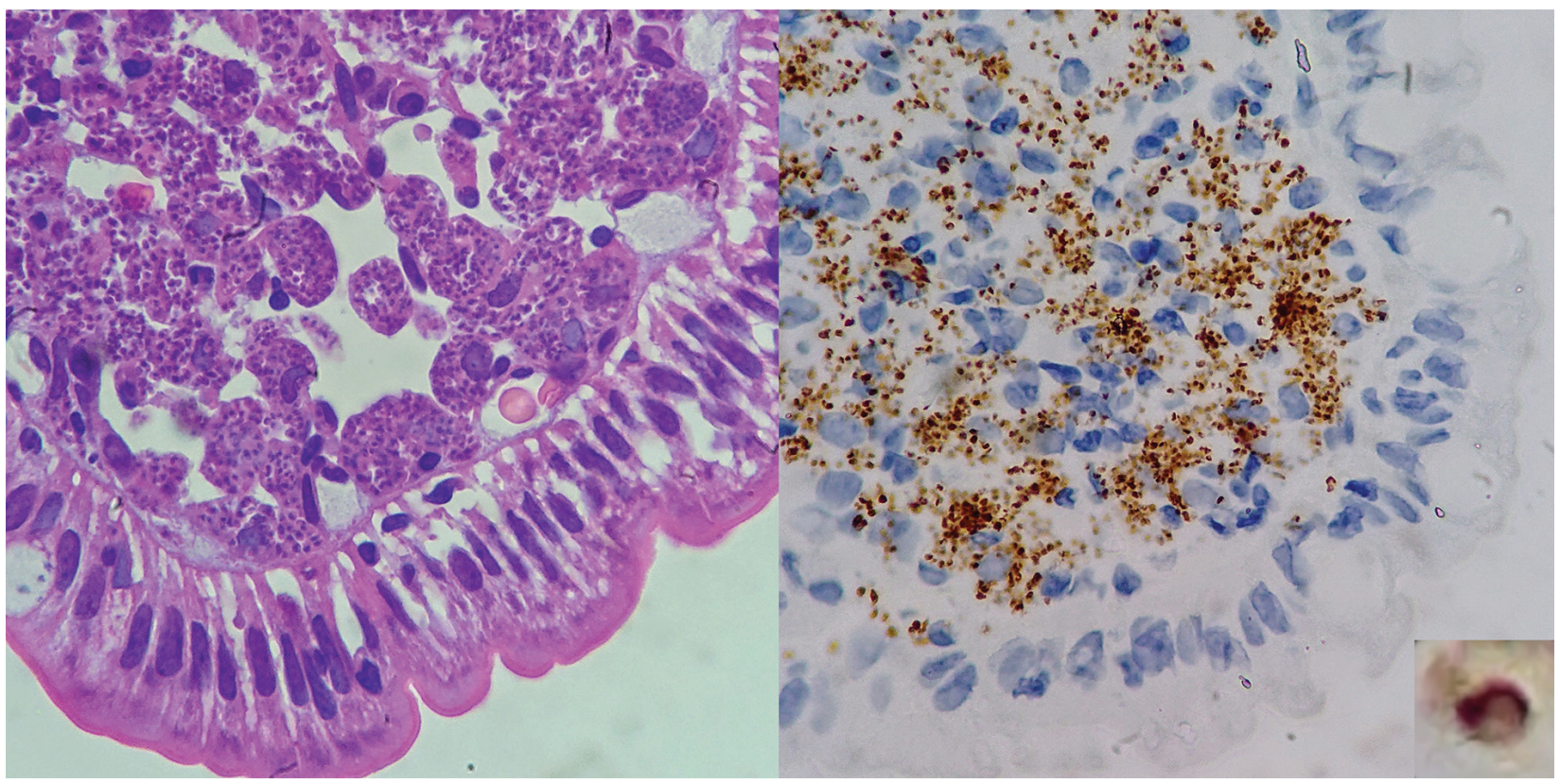

Figure 1 - Amastigotes within macrophages are observed on the left side, in a duodenal sample with a modified Ridley parasite index of $6(\mathrm{H} \& \mathrm{E}, 1000 \mathrm{x})$. A slide of this same sample is shown with the immunohistochemical reaction using the clone MTB1 of CD1a (1000x) on the right side. At the bottom, on the right corner, an amastigote is shown in detail, stained by immunohistochemistry in a pattern of peripheral membrane-like reinforcement, leaving the unstained nucleus in the center, and presenting with a reinforcement in one pole, apparently because of the kinetoplast staining (Digitally magnified. Original 1,000X magnification). 
Table 1 - Results divided by group and stratified according to the biopsy site and Modified Ridley Parasitic Index.

\begin{tabular}{|c|c|c|c|c|c|}
\hline Group & Biopsy Site & No. of cases & Ridley index & CD1a (O10) & CD1a (MTB1) \\
\hline \multirow{5}{*}{$\begin{array}{l}\text { True positives } \\
\text { (amastigotes) }\end{array}$} & Spleen & 1 & 2 & Negative & Negative \\
\hline & Duodenum & 3 & $4-6$ & 3 Negatives & 3 Positives \\
\hline & Liver & 2 & $2-3$ & $\begin{array}{l}1 \text { Positive } \\
1 \text { Negative }\end{array}$ & 2 Positives \\
\hline & Lymph node & 2 & 3 & 2 Negatives & 2 Positives \\
\hline & Bone Marrow & 5 & $1-3$ & 5 Negatives & $\begin{array}{l}2 \text { Positives } \\
3 \text { Negatives }\end{array}$ \\
\hline \multirow{2}{*}{$\begin{array}{l}\text { False positives } \\
\text { (cellular debris within } \\
\text { macrophages) }\end{array}$} & Lymph node & 1 & 0 & Negative & Negative \\
\hline & Bone Marrow & 1 & 0 & Negative & Negative \\
\hline \multirow{7}{*}{$\begin{array}{l}\text { True negatives } \\
\text { (Histoplasma } \\
\text { capsulatum yeast } \\
\text { cells) }\end{array}$} & Larynx & 1 & 2 & Negative & Negative \\
\hline & Lymph node & 2 & $2-3$ & 2 Negatives & 2 Negatives \\
\hline & Bone Marrow & 1 & 0 & Positive & Negative \\
\hline & Oral mucosa & 1 & 0 & Negative & Negative \\
\hline & Nasal mucosa & 1 & 2 & Negative & Negative \\
\hline & Skin & 1 & 2 & Positive & Negative \\
\hline & Lung & 1 & 0 & Negative & Negative \\
\hline \multirow{4}{*}{$\begin{array}{l}\text { False negatives } \\
\text { (negative biopsy, } \\
\text { positive myelogram } \\
\text { or K39 test) }\end{array}$} & Stomach & 1 & 0 & Negative & Negative \\
\hline & Liver & 1 & 0 & Negative & Negative \\
\hline & Lymph node & 2 & 0 & 2 Negatives & 2 Negatives \\
\hline & Bone Marrow & 21 & 0 & 21 Negatives & 21 Negatives \\
\hline
\end{tabular}

biopsies, we obtained, sensitivity of the MTB1 clone was $58.33 \%$, specificity $100 \%$ and accuracy $75 \%$.

In respect to the $\mathrm{O} 10$ clone, sensitivity was $8.33 \%$, specificity $87.50 \%$ and accuracy $40 \%$. These results are summarized in Table 2. Two specimens with Histoplasma capsulatum were positive for clone $\mathrm{O} 10$.

Table 2 - Results after the exclusion of bone marrow biopsies.

\begin{tabular}{lcc}
\hline & O10 & MTB1 \\
\hline Sensitivity & $8.33 \%$ & $58.33 \%$ \\
Specificity & $87.50 \%$ & $100 \%$ \\
Accuracy & $40.00 \%$ & $75 \%$ \\
\hline
\end{tabular}

\section{DISCUSSION}

Comparing the tests using MTB1 and O10 clones, there was greater sensitivity, specificity and, consequently, accuracy with the use of clone MTB1, corroborating the findings of other studies ${ }^{8,13}$. The immunohistochemical reactions using both clones were negative in the false positive samples, which shows that the use of immunohistochemistry may be relevant for the differential diagnosis between amastigotes present within macrophages and cellular debris.
The intermediate sensitivity obtained can be related to the fact that not all amastigotes will be phagocytized by CD1a positive cells. This antigenic exposure will only be possible in the papillary areas of the dermis where CD1a positive dendritic cells are found, so that amastigotes confined to deeper zones of the dermis will have lower chances of being exposed to CD1a. Because they multiply by binary fission, only amastigotes previously exposed to $\mathrm{CD} 1 \mathrm{a}$ will produce more $\mathrm{CD} 1 \mathrm{a}$ positive amastigotes ${ }^{8}$. Thus, one can infer that only amastigotes that descend from parasites previously exposed to CD1a in the skin will present positivity to this marker in internal organs.

In our study, there was a striking difference in sensitivity between the analysis using all cases and the one excluding the cases in which the bone marrow was the only site of biopsy. In the pathology laboratories of the participating institutions, nitric acid is used as a decalcifier in bone marrow biopsy specimens, which may have denatured CD1a in amastigotes and caused negative immunohistochemical reactions despite the presence of the parasite. The fact that this study is retrospective, using samples already processed with nitric acid, did not allow us to compare the effect of Ethylenediaminetetraacetic acid (EDTA), another decalcifying agent that seems to be less deleterious for 
immunohistochemical stainings ${ }^{14}$. Although none of the samples of Histoplasma capsulatum had positive results with the use of clone MTB1, 25\% of them had positive results with clone $\mathrm{O} 10$, which can lead to misdiagnosis. It is imperative to do perform special stainings for fungi, such as PAS and Grocott's methenamine silver, to avoid misdiagnosis.

We conclude that immunohistochemical testing with the CD1a antigen, clone MTB1, despite the intermediate sensitivity observed in this study, can be useful in the differential diagnosis of visceral leishmaniasis, helping to discriminate leishmania amastigotes from other pathogens with similar morphology and cellular debris in different samples, except in bone marrow biopsies previously decalcified with nitric acid.

\section{ACKNOWLEDGMENTS}

We thank the directors of the participating pathology laboratories, José Telmo Valença Júnior (Hospital Universitário Walter Cantídio) and André Luís Nunes Avelino (Instituto do Câncer do Ceará) for allowing this study. We also thank Diane Isabelle Magno Cavalcante for her guidance and Denyse Sales Veloso Albuquerque for her help in this article writing.

\section{REFERENCES}

1. Costa JM. Epidemiologia das Leishmanioses no Brasil. Gaz Med Bahia. 2005;75:3-17.

2. Brasil. Ministério da Saúde. Departamento de Vigilância Epidemiológica. Manual de vigilância e controle da Leishmaniose Visceral. Brasília: Ministério da Saúde; 2014. [cited 2018 Nov 27] Available from: http://bvsms.saude.gov.br/ bvs/publicacoes/manual_vigilancia_controle_leishmaniose_ visceral_1edicao.pdf
3. Ready P. Epidemiology of visceral leishmaniasis. Clin Epidemiol. 2014;6:147-54.

4. Lemos EM, Carvalho SF, Corey R, Dietze R. Avaliação do teste rápido utilizando o antígeno recombinante $\mathrm{k} 39$ no diagnóstico da leishmaniose visceral no Brasil. Rev Soc Bras Med Trop. 2003;36 Suppl 2:36-8.

5. Cotelingam JD. Bone marrow biopsy: interpretive guidelines for the surgical pathologist. Adv Anat Pathol. 2003;10:8-26.

6. Gayathri B, Rao K. Pancytopenia: a clinico hematological study. J Lab Physicians. 2011;3:15-20.

7. Guarner J, Brandt ME. Histopathologic diagnosis of fungal infections in the $21^{\text {st }}$ century. Clin Microbiol Rev. 2011;24:24780 .

8. Fernandez-Flores A, Rodriguez-Peralto JL. Morphological and immunohistochemical clues for the diagnosis of cutaneous leishmaniasis and the interpretation of CD1a status. J Am Acad Dermatol. 2016;74:536-43.

9. Coventry B, Heinzel S. CD1a in human cancers: a new role for an old molecule. Trends Immunol. 2004;25:242-8.

10. Karram S, Loya A, Hamam H, Habib RH, Khalifeh I. Transepidermal elimination in cutaneous leishmaniasis: a multiregional study. J Cutan Pathol. 2012;39:406-12.

11. Jabbour MN, Issa G, Charafeddine K, Simaan Y, Karam M, Khalifeh $\mathrm{H}$, et al. The immune microenvironment in cutaneous leishmaniasis. J Eur Acad Dermatol Venereol. 2015;29:1170-9.

12. Ridley DS, Ridley MJ. The evolution of the lesion in cutaneous leishmaniasis. J Pathol. 1983;141:83-96.

13. Sundharkrishnan L, North JP. Histopathologic features of cutaneous leishmaniasis and use of CD1a staining for amastigotes in Old World and New World leishmaniasis. J Cutan Pathol. 2017;44:1005-11.

14. Ramsay A, Pomplun S, Wilkins B. Tissue pathways for lymph node, spleen and bone marrow trephine biopsy specimens. London: The Royal College of Pathologists; 2017. [cited 2019 Apr 2]. Available from: https://www.rcpath.org/uploads/assets/ uploaded/1c9e6210-5b12-44a1-812091d307aa4cce.pdf 